\title{
Neurological manifestations in 404 COVID-19 patients in Washington State
}

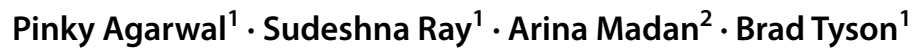

Received: 11 June 2020 / Revised: 12 July 2020 / Accepted: 14 July 2020 / Published online: 6 August 2020

c) Springer-Verlag GmbH Germany, part of Springer Nature 2020

\section{Dear Sirs,}

We report neurological manifestations in 404 consecutive patients with COVID-19 seen in outpatient clinics and hospitalized between February 20, 2020 and May 4, 2020 at Evergreen Hospital, Kirkland, Washington, USA. This hospital was among the first to report cases and deaths of COVID-19 patients in the United States. COVID-19 is caused by the severe acute respiratory syndrome coronavirus 2 (SARS-CoV-2). Most neurological manifestations of COVID-19 have been reported from non-USA health systems for hospitalized patients [1-3].

All patients had laboratory confirmed SARS-CoV-2 infection. Only new onset neurological manifestations were included. Study data were extracted from electronic medical records, and all neurological manifestations were confirmed via chart review by a neurologist and neuropsychologist. The study was approved by institutional review board and ethics committee. Core presenting symptoms were fever, cough, and shortness of breath.

Clinical characteristics and admission laboratory measures are summarized in Table 1. Shapiro's test revealed significant deviations from normal distribution. Therefore, non-parametric alternatives to the $t$ test (Wilcox rank sum test and Hodges-Lehmann estimation) were used, at alpha level of 0.05 , adjusted for multiple comparisons using the Benjamini-Hochberg method.

Female patients younger than 65 years old were more likely to have neurological symptoms; however, the findings were not statistically significant. Asian patients were 2.02 times more likely to have neurological symptoms than

Pinky Agarwal

pagarwal@evergreenhealth.com

1 Evergreen Health Neuroscience Institute, Kirkland, WA, USA

2 University Hospitals Birmingham NHS Foundation Trust, Birmingham, UK
Caucasian patients, but the findings did not meet statistical significance either.

Frequencies of neurological symptoms are summarized in Table 2. Neurological findings were reported in 295 of $404(73.0 \%)$ patients, which is higher than reported by the previous studies from China and Spain [1,3]. Mao et al. reported neurological manifestations in $36.4 \%$ of a sample of hospitalized patients from Wuhan, China [1] and RomeroSánchez et al. reported neurological manifestations in 57.4\% of a sample of hospitalized COVID-19 patients in Spain [3]. Helms et al. reported neurological signs in $84 \%$ of hospitalized patients in France; however, this was in a subset of patients with severe SARS-CoV-2 infection in intensive care, frequently under general anesthesia [2].

In our study, 208 of 404 (51.5\%) patients presented with Central Nervous System (CNS) symptoms. The most prevalent CNS symptoms were altered mental status $(86,21.3 \%)$, headache $(82,20.3 \%)$, and dizziness $(31,7.7 \%)$. Of the 86 patients with altered metal status, $49(57.0 \%)$ had pre-existing dementia. Out of 404 patients, 163 (40.3\%) presented with Peripheral Nervous System (PNS) symptoms. The most prevalent PNS symptoms were myalgia $(131,32.4 \%)$ and impairment of taste $(27,6.7 \%)$ and smell $(18,4.5 \%)$.

Acute neurological manifestations were seen in 99 of 404 (24.5\%) COVID-19 patients with altered mental status seen in $86(21.3 \%)$, critical illness myopathy in $8(2.0 \%)$, stroke including one hemorrhagic stroke in $3(0.7 \%)$, and seizures in $2(0.5 \%)$. Six patients had multiple acute neurological manifestations.

This study is useful to inform clinicians treating COVID19 patients about neurological manifestations, which may be overlooked due to life-threatening acute respiratory complications. The common occurrence of neurological manifestations in outpatient clinics is important to recognize as being related to COVID-19 to initiate early intervention and prevent community spread. Altered mental status may be the presenting symptom of COVID-19, particularly in patients with pre-existing dementia. 
Table 1 Clinical characteristics and admission lab measures of all COVID-19 patients

\begin{tabular}{|c|c|c|c|c|c|}
\hline \multirow[t]{2}{*}{ Characteristics } & $\begin{array}{l}\text { Without neurological symp- } \\
\text { toms (109 patients) }\end{array}$ & $\begin{array}{l}\text { With neurological symp- } \\
\text { toms ( } 295 \text { patients) }\end{array}$ & \multirow[t]{2}{*}{$p$ values } & \multirow{2}{*}{\multicolumn{2}{|c|}{ Odds ratio }} \\
\hline & No. $(\%)$ of patients & No. (\%) of patients & & & \\
\hline \multicolumn{6}{|l|}{ Age, year ${ }^{a}$} \\
\hline$\geq 65$ & $52(12.9)$ & $129(31.9)$ & \multirow[t]{2}{*}{0.55} & \multicolumn{2}{|c|}{$1.17(0.75,1.82)$} \\
\hline$<65$ & $57(14.1)$ & $166(41.1)$ & & \\
\hline \multicolumn{6}{|l|}{ Gender } \\
\hline Male & $48(11.9)$ & $149(36.9)$ & \multirow[t]{2}{*}{0.30} & \multicolumn{2}{|c|}{$0.77(0.50,1.20)$} \\
\hline Female & $61(15.1)$ & $146(36.1)$ & & & \\
\hline \multicolumn{6}{|l|}{ Race } \\
\hline Caucasian & $93(23.0)$ & $237(58.7)$ & \multirow[t]{6}{*}{0.18} & \multicolumn{2}{|c|}{ NA } \\
\hline African American & $5(1.2)$ & $6(1.5)$ & & \multicolumn{2}{|c|}{$0.39(0.14,1.48)$} \\
\hline Asian & $5(1.2)$ & $31(7.7)$ & & \multicolumn{2}{|c|}{$2.02(0.88,5.75)$} \\
\hline Native Hawaiian or Pacific Islander & $0(0.0)$ & $4(1.0)$ & & \multicolumn{2}{|c|}{$1.56(0.19,66.5)$} \\
\hline American Indian or Alaska Native & $0(0.0)$ & $3(0.7)$ & & \multicolumn{2}{|c|}{$1.17(0.14,53.87)$} \\
\hline Undetermined & $6(1.5)$ & $14(3.5)$ & & \multicolumn{2}{|c|}{$0.78(0.34,2.28)$} \\
\hline \multicolumn{6}{|l|}{ Core symptoms } \\
\hline Fever $^{\mathrm{b}}$ & $60(14.9)$ & $194(48.0)$ & \multirow[t]{3}{*}{0.75} & \multicolumn{2}{|c|}{ NA } \\
\hline Cough & $76(18.8)$ & $212(52.5)$ & & \multicolumn{2}{|c|}{$0.86(0.58,1.27)$} \\
\hline Shortness of breath & $57(14.1)$ & $166(41.1)$ & & \multicolumn{2}{|c|}{$0.90(0.59,1.37)$} \\
\hline Preadmission comorbidities & & & & & \\
\hline Cardiovascular disease ${ }^{c}$ & $31(7.7)$ & $63(15.6)$ & 0.02 & NA & \\
\hline Peripheral vascular disease & $1(0.2)$ & $21(5.2)$ & & & $(1.29,39.3)$ \\
\hline Dementia $^{c}$ & $21(5.2)$ & $34(8.4)$ & & & $(0.40,1.58)$ \\
\hline $\mathrm{COPD}^{\mathrm{c}}$ & $17(4.2)$ & $41(10.1)$ & & & $(0.58,2.38)$ \\
\hline Diabetes mellitus ${ }^{c}$ & $12(3.0)$ & $48(11.9)$ & & & $(0.91,4.09)$ \\
\hline Chronic kidney disease & $14(3.5)$ & $36(8.9)$ & & & $(0.59,2.63)$ \\
\hline Asthma & $8(2.0)$ & $17(4.2)$ & & & $(0.41,2.57)$ \\
\hline Cancer & $4(1.0)$ & $17(4.2)$ & & & $(0.63,5.92)$ \\
\hline Hypertension $^{\mathrm{c}}$ & $16(4.0)$ & $52(12.9)$ & & & $(0.78,3.17)$ \\
\hline Rheumatoid arthritis & $6(1.5)$ & $4(1.0)$ & & & $(0.10,1.23)$ \\
\hline Others & $1(0.2)$ & $10(2.5)$ & & & $(0.60,20.3)$ \\
\hline Admission lab measures, median (ran & & & & & \\
\hline & $\begin{array}{l}\text { Without neurological } \\
\text { symptoms median (inter- } \\
\text { quartile range) }\end{array}$ & $\begin{array}{l}\text { With neurological symp- } \\
\text { toms median (interquartile } \\
\text { range) }\end{array}$ & Confidence intervals & $p$ value & Reference range \\
\hline White blood cell count $/ \mu \mathrm{L}$ & $7630(5815-11,710)$ & $6470(4935-9680)$ & -2050.00 to 269.99 & 0.48 & $4000-9000$ \\
\hline Absolute neutrophil count/ $\mu \mathrm{L}$ & $4910(4090-7950)$ & $4730(3320-7895)$ & -1500.00 to 389.99 & & $1780-5380$ \\
\hline Absolute lymphocyte count/ $\mu \mathrm{L}$ & $960(710-1260)$ & $990(747.5-1315)$ & -129.99 to 190.00 & 0.92 & $1320-3570$ \\
\hline Absolute platelet $10^{3} / \mu \mathrm{L}$ & $223(168-295.5)$ & $204(154-262)$ & -46.99 to 12.99 & 0.53 & $163-337$ \\
\hline C-reactive protein, $\mathrm{mg} / \mathrm{dL}$ & $9.82(3.21-15.02)$ & $8.02(4.82-13.04)$ & -3.38 to 3.51 & 0.92 & $\leq 01.50$ \\
\hline Lactate dehydrogenase(U/L) & $314(281.25-414.75)$ & $366.5(343.75-457.25)$ & -39.99 to 163.99 & 0.48 & $100-200$ \\
\hline Aspartate aminotransferase, U/L & $36(25.5,56)$ & $34(25,51)$ & -7.99 to 5.00 & 0.92 & $5-40$ \\
\hline Alanine aminotransferase, U/L & $24(15.5,47.5)$ & $25(17,40.75)$ & -6.00 to 5.00 & 0.92 & $5-50$ \\
\hline Blood urea nitrogen, $\mathrm{mmol} / \mathrm{L}$ & $15(9.5,27)$ & $19(13,30)$ & 0.00 to 6.99 & 0.48 & $7-23$ \\
\hline Creatinine, $\mu \mathrm{mol} / \mathrm{L}$ & $0.9(0.7-1.25)$ & $1.0(0.8,1.3)$ & -0.00 to 0.20 & 0.50 & $0.7-1.5$ \\
\hline Creatine kinase, $\mathrm{U} / \mathrm{L}$ & $108(76-184)$ & $111(65,224)$ & -37.99 to 37.99 & 0.92 & $35-232$ \\
\hline
\end{tabular}

${ }^{\mathrm{a}}$ The median age of the patients was 61 years

${ }^{\mathrm{b}}$ Patient complaint of fever

${ }^{\mathrm{c}}$ The most common comorbid conditions 
Table 2 Frequency of the neurological symptoms in COVID-19 patients

Nervous system symptoms

\begin{tabular}{lc}
\hline All & $295(73 \%)$ \\
CNS $^{\mathrm{a}}$ & $208(51.5 \%)$ \\
Dizziness & $31(7.7 \%)$ \\
Headache & $82(20.3 \%)$ \\
Altered mental status & $86(21.3 \%)$ \\
Acute cerebrovascular disease (Stroke) & $3(0.7 \%)$ \\
Gait imbalance & $20(5.0 \%)$ \\
Seizure & $2(0.5 \%)$ \\
PNS & $163(40.3 \%)$ \\
Taste impairment & $27(6.7 \%)$ \\
Smell impairment & $18(4.5 \%)$ \\
Vision impairment & $5(1.2 \%)$ \\
Nerve pain & $1(0.2 \%)$ \\
Myalgia & $131(32.4 \%)$ \\
Rhabdomyolysis & $4(1.0 \%)$ \\
Critical illness myopathy & $8(2.0 \%)$ \\
\hline
\end{tabular}

${ }^{a}$ More than one clinical symptom was present in $61 \mathrm{CNS}$ and 21 PNS patients

\section{Compliance with ethical standards}

Conflicts of interest The authors report no conflict of interest.

Ethical standard statement Study is in compliance with ethical standards.

\section{References}

1. Mao M, Jin H, Wang M et al (2020) Neurological manifestations of hospital patients with Coronavirus disease 2019 in Wuhan, China. JAMA Neurol 77(6):683-690

2. Helms J, Kremer S, Merdji H et al (2020) Neurological features in severe SARS-CoV-2 infection. N Engl J Med 382:2268-2270

3. Romero-Sánchez CM, Díaz-Maroto I et al (2020) Neurologic manifestations in hospitalized patients with COVID-19: the ALBACOVID Registry. Neurology. https://doi.org/10.1212/WNL.00000 00000009937 